\title{
Kokori, un Serious Game. La perspectiva de los estudiantes ante una propuesta de aprendizaje innovadora.
}

\author{
Kokori a Serious Game. The Prospect of Students to a Proposal for \\ Innovative Learning.
}

\author{
María Luisa Bossolasco \\ Universidad Nacional de Tucumán. Argentina. \\ mlbossolasco@gmail.com \\ Roxana Judith Enrico \\ Universidad Nacional de Tucumán. Argentina. \\ roxyenrico@gmail.com \\ Beatríz Adriana Casanova \\ Universidad Nacional de Tucumán. Argentina \\ bettinacasanova@gmail.com \\ Eugenia Elizabeth Enrico \\ Universidad Nacional de Tucumán. Argentina. \\ eugenrico@gmail.com
}

\section{Resumen}

Se presentan aquí los resultados de una experiencia de aprendizaje en la cual se propuso a los alumnos participar de un videojuego, como una actividad más en su proceso de aprender. El propósito del estudio fue indagar las razones por la cual los alumnos eligieron jugar/no jugar así como sus creencias sobre los posibles beneficios para el aprendizaje. El análisis de los resultados se realizó poniendo el foco en los procesos motivacionales.

Participaron de la experiencia alumnos que cursan el primer año del nivel superior, en carreras del área de Ciencias Biológicas. Los resultados pusieron de manifiesto que la participación en la actividad de jugar el videojuego parece haber respondido a metas extrínsecas, pero al indagar luego sobre la experiencia de aprendizaje, parecería que la misma ha generado una posterior motivación intrínseca. Jugaron para ganar un evaluativo, pero luego de haber vivido la experiencia de aprendizaje, la incorporaron como vivencia significativa y viable de replicar en otros contextos de aprendizaje.

En la discusión se hace referencia a aquellas cuestiones del entorno que podrían actuar como favorecedoras de la motivación, poniendo especial atención al diseño de las actividades académicas.

\footnotetext{
Abstract

This article shows the results of a learning experience which was proposed to the students to participate in a video game, considering this game as another activity in their learning process. The purpose of the study was to investigate the reasons why students chose to play / not to play as well as their beliefs about the feasibility of including such proposals in education and its potential benefits for learning. The analysis of the results was performed by focusing on motivational processes.

First year students and teachers of the Biological Sciences degree took part in this experience. The results showed that the participation in the activity of playing the video game seems to have responded to extrinsic goals, but then by investigating the learning
} 
experience and the ability to replicate this type of academic activities in other contexts, it would apparently have generated a subsequent intrinsic motivation. The students came to play to take an evaluative exercise, but after having the experience of learning, it was incorporated as a significant and applicable experience in other learning contexts.

Those environmental issues that could act as motivational elements are referred in the discussion, paying special attention to the design of academic activities.

\section{Palabras claves}

Videojuegos serios, Enseñanza, Aprendizaje, Motivación, Innovación pedagógica.

\section{Key words}

Serious games, Teaching, Learning, Motivation, Educational innovation.

\section{Introducción}

En el presente trabajo se ofrece una primera aproximación a la visión de un grupo de alumnos de nivel superior universitario, que formaron parte de una experiencia de aprendizaje en donde se les propuso participar de un videojuego. Dicho videojuego se enmarca dentro de los conocidos como serious games, en el cual se abordan contenidos trabajados previamente en la materia. El propósito del estudio es indagar las razones por la cual los alumnos eligieron jugar/no jugar así como sus creencias sobre la factibilidad de incluir este tipo de propuestas en la enseñanza y sus posibles beneficios para el proceso de aprendizaje.

Cuando se piensa en la inclusión de videojuegos en la enseñanza, diferentes autores argumentan en su favor, señalando que este tipo de propuestas incide de manera favorable en la motivación de los alumnos, lo cual redundaría positivamente en el progreso del aprendizaje. En función de ello la interpretación de las respuestas de los alumnos, se realiza poniendo el foco en los procesos motivacionales vinculados al aprendizaje.

\section{Objetivos}

1. Indagar acerca de las razones por la cual los alumnos eligieron jugar/no jugar un videojuego educativo.

2. Identificar creencias acerca de la factibilidad de incluir este tipo de actividades en la enseñanza.

3. Identificar procesos motivacionales subyacentes en las respuestas de los alumnos participantes.

\section{Marco Teórico}

Castells (2003), Ito (2010) y Gardner (2014), entre otros, llaman la atención sobre el modo en que los medios digitales, el acceso a la web y a las redes sociales que en ella se generan, han reconfigurado los contextos de comunicación, de expresión personal y de juego generando una nueva ecología cultural y social. Los teléfonos celulares, las apps, los sitios en los que se comparten fotos y videos, los juegos en red, construyen esta ecología; la cual tiene en el centro las prácticas que llevan adelante los jóvenes con estos nuevos medios. Para estos autores, existe una nueva manera de ser joven, en el contexto de estas interacciones.

Con la tendencia actual a masificarse el acceso y uso de dispositivos tecnológicos entre los jóvenes y la disposición a incluir recursos proveniente del área de las TIC desde 
lineamientos de políticas educativas nacionales, en nuestro país ${ }^{1}$, se sostiene la necesidad de acortar la distancia entre lo que los alumnos hacen y saben hacer con estos dispositivos fuera de los contextos educativos y lo que efectivamente hacen dentro de la escuela. A esta distancia Buckingham (2007) la denomina segunda brecha, refiriéndose a la diferencia que existe "entre las prácticas y lenguajes que los chicos desarrollan fuera de la escuela y las que se ofrecen dentro de ella" (Buckingham, 2007, p. 15)

Dentro del abanico de recursos y posibilidades que ofrecen las nuevas tecnologías, los videojuegos suelen aparecer en el discurso de los adultos, padres y educadores, como reñidos con la enseñanza y el aprendizaje. En general se los asocia con el ocio y el tiempo libre y aparecen muy vinculados con efectos nocivos en aspectos como la socialización, la lectura, la reflexión, la comprensión de conceptos; saberes que se privilegian en la escolarización formal.

Ante esta situación resulta necesario señalar que en la actualidad existe el desarrollo de videojuegos, diseñados de manera específica con fines educativos. En este sentido, se puede encontrar una vasta clasificación de videojuegos en función de diversos criterios. En el presente trabajo nos interesa focalizar en aquellos juegos denominados serious games (juegos serios), que son aquellos juegos que además del efecto lúdico inherente al género, fueron diseñados con un propósito específico más allá del entretenimiento. Este tipo de juegos transmiten mensajes o ideas sobre determinado tema, buscan intencionalmente generar habilidades, reacciones, interpelar a los jugadores, contribuir a internalizar conceptos.

Actualmente los serious games atraen interés desde distintos ámbitos: educación, formación profesional, asistencia médica, trabajo en equipo, publicidad, políticas públicas; entre otros. En el ámbito específico de la educación parecería existir aún una tensión no resuelta entre contenido y forma. En este sentido, Segal y otros (2012) señalan que aún existe cierta disyuntiva en el diseño de videojuegos, entre el contenido que se espera que el juego dispare y ayude a construir y la necesidad de no traicionar al jugador ofreciéndole un artefacto digital que enseña, pero que ha dejado de ser un juego. O por el contrario, un videojuego centrado en los recursos clásicos del género (humor, jugabilidad, velocidad, sonido, interface), pero que no añade ningún aporte a la aproximación de un contenido que se podría ofrecer sin un videojuego. Si fuese este el caso, el videojuego pasaría a formar parte sólo de una motivación externa que entretiene y divierte, ayudando a captar la atención de los alumnos, pero que no aporta sustantivamente a la construcción de ideas o a la posibilidad de generar debates entre los participantes.

Revuelta y Guerra (2012) señalan que el uso de los videojuegos como herramienta didáctica y el interés por los mismos, ha quedado plasmado en el $1^{\circ}$ Congreso Internacional de Videojuegos y Educación (CIVE 12) celebrado en Alicante, España; en donde docentes y creadores de videojuegos expusieron su visión de los mismos como elemento con posibilidades lúdico-educativas para la adquisición de habilidades y nuevos saberes en el campo educativo.

Quienes argumentan a favor de introducir este tipo de recursos en el proceso de enseñanza-aprendizaje, sostienen que son propuestas que resultan motivadoras para los participantes. En este sentido, la motivación -pre requisitos para que tenga lugar el aprendizaje- parecería ser un elemento clave para justificar su incorporación en el diseño de una propuesta didáctica. Jugar es una experiencia atractiva y motivadora,

\footnotetext{
${ }^{1}$ Programa Nacional "Conectar Igualdad", creado en abril de 2010 a través del Decreto No 459/10.
} 
capta la atención, estimula la flexibilidad del pensamiento para saber buscar y adoptar nuevas estrategias frente a cualquier situación que se presente, por compleja o difícil que esta parezca.

Es vasta la investigación en el área de la psicología educacional en donde se estudia acerca de los factores personales - tanto cognitivos como motivacionales- que interactúan con las características del contexto de la clase, en el aprendizaje de las personas. En este sentido y poniendo foco en los aspectos motivacionales relacionados con el aprendizaje, Paoloni y otros (2006) señalan que en los estudios sobre motivación académica han comenzado a prevalecer aquellos enfoques en donde tanto los aspectos cognitivos como los motivacionales son esenciales para comprender el compromiso del estudiante para con su aprendizaje; y señalan además que ambos aspectos personales deben analizarse desde una aproximación situada, es decir, una mirada en donde se considere al contexto como co-constructor del proceso motivacional de los estudiantes.

Ampliando esta idea, en un estudio reciente Chiecher, Paoloni y Ficco (2014) destacan la importancia de tomar en consideración ciertas variables que, desde el contexto, los docentes involucrados en el proceso de enseñanza-aprendizaje podrían manejar, incidiendo a favor de la motivación de los alumnos. En el diseño de los entornos de enseñanza-aprendizaje se ponen en juego múltiples variables (Bossolasco, 2013) que deberían explicitarse para poder pensar en primer lugar que qué y el para qué de la propuesta que se presenta, para pensar luego en el cómo y con qué. Al modificar ciertas variables de esos entornos, se podría incidir en los estilos motivacionales de los participantes.

Paoloni y otros (2006) retoman el concepto de motivación propuesto por Huertas (1997) quien la considera como "un proceso psicológico dinámico y complejo que determina la planificación y actuación del sujeto en función a metas previamente establecidas." (Huertas, 1997, citado en Paoloni y otros, 2006, pp. 36) Partiendo de los planteos de Pintrich, Smith, García Y McKeachie (1991) Paoloni y otros identifican una serie de variables que jugarían un papel relevante en los procesos motivacionales de los estudiantes. Algunas de estas variables estarían haciendo referencia a factores personales y otras a ciertos aspectos contextuales.

Entre las variables personales que estarían afectando la motivación de los estudiantes Paoloni y otros (2006) identifican las siguientes:

- Orientación hacia las metas: Diferentes autores consideran que el establecimiento de metas es un importante proceso cognitivo-social que influye en la motivación de los estudiantes. Se podrían identificar dos orientaciones básicas hacia la meta; una relacionada con metas intrínsecas, centradas en el proceso de aprendizaje en sí y orientadas al control de la tarea y otras llamadas metas extrínsecas, que se orientan hacia los resultados. Mientras las primeras se caracterizan por un interés en incrementar el conocimiento y mejorar la comprensión de un tema, las segundas reflejan un interés centrado en evitar una valoración negativa sobre el propio desempeño o un interés por demostrar que la capacidad de uno es superior a la de los demás. Los autores señalan que quienes se proponen metas del primer tipo seleccionan problemas de dificultad moderada, y emplean estrategias de aprendizaje más efectivas, mientras que quienes guían su aprendizaje hacia metas extrínsecas procuran maximizar la recompensa extrínseca con un mínimo de esfuerzo invertido.

- Creencias de autoeficacia: Los autores entienden a esta creencia como los juicios que tiene una persona acerca de la capacidad para desempeñarse en

Kokori, un serious game. La perspectiva de los estudiantes ante una propuesta de aprendizaje innovadora. 
acciones específicas, a la luz de metas específicas. Cuando mayor es la eficacia percibida, más desafiante será la meta que la persona establezca para sí y más firme el esfuerzo, persistencia y compromiso para con la tarea.

- Expectativa de resultados y valoraciones: Esta variable se encontraría muy vinculada a la anterior. La expectativa de resultado refiere a la estimación que hace la persona de que la conducta, una vez realizada, tendrá un resultado concreto. La valoración, por su parte, refiere a la percepción que tiene la persona de cuán interesante, importante o útil será para él la tarea o el resultado a alcanzar. Desde estos planteos se sugiere que las personas no se motivan por alcanzar metas consideradas imposibles ni por logra resultados no valorados.

- Atribuciones causales: Es el proceso por el cual una persona busca y obtiene explicaciones de los resultados conseguidos luego de una actuación determinada. Paoloni y otros (2006) señalan que según resultados de investigaciones habría estilos atribucionales diferentes. Por un lado existirían atribuciones internas, utilizado por aquellos alumnos que tienden a explicar los resultados en términos de habilidad, esfuerzo invertido, estrategia adoptada. Por el contrario las atribuciones externas son utilizadas por aquellos estudiantes que tienden a responsabilizar a factores externos por sus logros escolares, como la suerte, la ayuda del profesor, el tipo de tarea, etc.

- Creencias de control: Esta creencia sería determinante de la motivación intrínseca. Refiere a la sensación de que uno es responsable de sus acciones, que las inicia y las controla. Según señalan Paoloni y otros (2006) si se les da a los estudiantes la posibilidad de elegir, esto afecta positivamente la motivación intrínseca, favoreciendo una manera más animada y optimista de enfrentar la tarea. El contar con mayores niveles de autonomía promovería una motivación intrínseca, teniendo el alumno la posibilidad de manejar los recursos de aprendizaje, el manejo del tiempo, las búsquedas de ayuda, controlar sus comportamientos y tener un mayor control del ambiente.

Por su parte, entre las variables contextuales que podrían incidir sobre la motivación académica de los aprendices, señalan las siguientes:

- Diseño de las tareas y actividades de aprendizaje: Paoloni y otros (2006) señalan que entre las variables contextuales que influyen en la motivación de los estudiantes, el tipo de tarea que se le presente a los estudiantes, es de fundamental importancia. Se señalan ciertas características de las tareas que promoverían la motivación intrínseca, entre las que se mencionan:

$\checkmark$ La variedad y diversidad de las tareas presentadas; lo cual ayudaría a mantener el interés.

$\checkmark$ La percepción del valor de utilidad de una tarea (funcionalidad y significatividad), para el logro de metas futuras.

$\checkmark$ El nivel de dificultad debe ser acorde respecto a las posibilidades de aprendizaje de los alumnos.

$\checkmark$ El generar curiosidad en los estudiantes, tareas que resulten novedosas e imprevisibles, promovería conductas de exploración y motivación intrínseca. 
Implicar a los estudiantes en la fantasía y la ilusión de hacer algo a través de la simulación o juegos; aunque no demasiado exóticas porque pueden distraer la atención.

$\checkmark$ La colaboración y la interacción social en grupos, parecerían contribuir a elevar los niveles de motivación.

- Prácticas de evaluación, recompensas y feedback: Ames, 1992, citado en Paoloni y otros (2006) considera que si las prácticas de evaluación fomentan la comparación social, es probable que favorezcan una orientación hacia metas de desempeño. Para fomentar metas orientadas a los aprendizajes deberían proveer a los estudiantes de información sustantiva acerca de sus propias competencias y señalamientos sobe cómo mejorar dicho desempeño a futuro. Según señala Huertas, 1997, citado en Paoloni y otros (2006) las recompensan extrínsecas parecerían tener una veta negativa y otra positiva sobre la motivación. Parecerían ser positivas para generar metas intrínsecas en aquellos casos en que la actividad no produce inicialmente interés intrínseco en el alumno. En caso contrario, si el interés existe, proponer recompensas para tareas que de por sí resultan interesantes, podría disminuir la motivación intrínseca. En relación al feedback que el docente puede realizar, los autores anteriormente señalados sostienen que el feedback puede incrementar la motivación intrínseca y la autoeficacia percibida cuando se comunica a los alumnos que el error es parte del aprendizaje y cuando se señalan los progresos que se van haciendo en pos de alcanzar la meta propuesta. Al mismo tiempo señalan que el feedback negativo socavaría la motivación intrínseca.

- Distribución de la autoridad y la responsabilidad: Esta variable estaría relacionada con el sentimiento de control. Según los planteos de Ames, 1992, citado en Paoloni y otros (2006) proveer algo de control y posibilidades de elección dentro del contexto de la clase, incrementaría el interés por la tarea y el compromiso cognitivo con la misma, ya que contribuiría al desarrollo de motivación intrínseca.

\section{Descripción del juego}

Kokori $^{2}$, que significa "juego colectivo" en rapanui, la lengua originaria de la Isla de Pascua -Chile-, es el resultado de un Proyecto TIC Edu del Fondo de Fomento al Desarrollo Científico y Tecnológico (FONDEF) de Chile y surge del sueño de unos jóvenes que imaginaban una herramienta pedagógica que permitiera a los estudiantes aprender sobre la célula en un entorno de tres dimensiones, observando la estructura celular desde adentro, sus componentes de forma integrada, las relaciones de tamaños y funcionamiento.

Es un videojuego 3D de estrategia en tiempo real con el objetivo de resolver problemas o daños que se presentan en una célula animal. Estos objetivos se cumplen durante siete misiones, donde los estudiantes podrán conocer, recorrer e intervenir diferentes componentes de una célula lo que, se supone, les facilitará un aprendizaje entretenido, desafiante y multimedial. Contiene además un navegador de célula $3 \mathrm{D}$ con el que se puede recorrer el interior celular como un explorador de las estructuras y procesos

\footnotetext{
${ }^{2}$ Sitio oficial de Kokori: http://www.kokori.cl/
}

Kokori, un serious game. La perspectiva de los estudiantes ante una propuesta de aprendizaje innovadora. M.L. Bossolasco et al. 
celulares, sin necesidad de interactuar ni cumplir misiones, pensado para ser mostrado en el aula utilizando un proyector.

Kokori está diseñado para su aplicación en docencia por lo que presenta manuales y tutoriales para docentes y usuarios.

\section{El contexto de la experiencia}

La propuesta de jugar en este videojuego se implementó en la asignatura Biología Celular y de los Microorganismos de la Facultad de Ciencias Naturales e IML ${ }^{3}$, UNT (Universidad Nacional de Tucumán), en los años 2013 y 2014.

Esta asignatura de primer año, con una matrícula inicial de entre 300 y 400 alumnos, se dicta en el primer cuatrimestre y pertenece al ciclo básico de las Carreras de Licenciatura y Profesorado en Ciencias Biológicas con una modalidad de dictado teórica-práctica, con clases en aula y otras en laboratorio.

En las clases teórico-prácticas de aula se dictan temas teóricos en forma expositiva para todos los alumnos vinculando todos los temas del programa y su desarrollo práctico, realizando una articulación permanente para favorecer el aprendizaje.

En las clases teórico-prácticas de laboratorio se desarrollan los diferentes prácticos de una Guía de Trabajos Prácticos. Las mismas se dictan separando los alumnos en comisiones de 35 a 40 alumnos. Dentro del laboratorio, cada comisión se subdivide en grupos de trabajo de 7 a 8 alumnos, con un ayudante a cargo, para personalizar la enseñanza. En estas instancias los alumnos trabajan con material biológico recolectado en vivo o cultivado en la cátedra.

Para regularizar la asignatura se deben aprobar dos parciales, y para tener derecho a rendir el parcial se debe aprobar un porcentaje de los evaluativos parciales de cada tema.

En el desarrollo de cada tema se plantean ejemplos tangibles y organismos tipos, que los induzcan a comprender y asimilar los conceptos recibidos. Se emplean videos en caso de ser necesarios como recursos didácticos de apoyo a la enseñanza, además de imágenes y fotografías, presentaciones confeccionadas con Powerpoint o Prezi, maquetas y esquemas en afiches y posters. Todos estos recursos, parte de ellos disponibles en el aula virtual de la cátedra, sirven para ayudar al alumno a generar el poder de abstracción sobre lo "micro" y favorecer la compresión y el aprendizaje significativo.

Desde el año 2009, se habilitó un aula virtual en apoyo a la enseñanza presencial. Este entorno virtual constituye un entorno para complementar el cursado, extendiendo el trabajo que se lleva a cabo en el aula presencial y procurando que las clases resulten más claras, completas, atractivas y estimulantes. Además, los estudiantes encuentran un espacio de interacción que permite mantener el contacto con los docentes y entre pares, superando las barreras de las distancias físicas y temporales. También se desarrollan diferentes actividades virtuales (situaciones problemáticas, controles de lectura y autoevaluaciones virtuales) que están pensadas para abarcar las múltiples inteligencias del numeroso grupo de alumnos.

Con estas experiencias en el dictado de la materia, y luego de detectar falencias en la comprensión y abstracción de la estructura celular en forma reiterada, en las diferentes

\footnotetext{
${ }^{3}$ IML: Instituto Miguel Lillo. Instituto que forma parte de la Facultad de Ciencias Naturales en la Universidad Nacional de Tucumán.
} 
cohortes de alumnos, se decidió experimentar con nuevos materiales didácticos digitales. La implementación de estos materiales significó un proceso evolutivo que comenzó con la inclusión del aula virtual y continuó incorporando actividades y materiales de aprendizajes interactivos y en una modalidad de trabajo colaborativo; mudando desde una modalidad netamente presencial a una modalidad mixta.

En este contexto y con el objetivo de ofrecer a los alumnos una instancia de aprendizaje de la estructura y el funcionamiento celular a través de la interacción con un material de simulación, se ofrece a los alumnos participar del juego Kokori.

Esta actividad no fue obligatoria sin embargo, para promover que un mayor número de alumnos se interesara por pasar la experiencia, se ofreció a los alumnos la posibilidad de computar como un evaluativo aprobado a los cincuenta puntajes más altos. Para ello, los alumnos debieron publicar la captura de su pantalla con el puntaje obtenido en las diferentes misiones, dentro del aula virtual de la cátedra, en un foro habilitado para ese fin.

Con esta actividad no se buscaba evaluar aprendizajes efectivos, por parte de los alumnos, sobre los contenidos de células trabajados en la unidad; sino ofrecerles un recurso más que permitiera promover aprendizajes. Participaron de esta experiencia un total de 110 alumnos. Tal como se señaló fue una participación voluntaria. La actividad no fue realizada en el contexto de la clase, sino que se propuso como una tarea a realizar fuera del horario de cursado presencial.

A partir de la experiencia, y con el propósito de conocer las razones por la cual los alumnos eligieron jugar/no jugar un videojuego educativo, identificar creencias acerca de la factibilidad de incluir este tipo de actividades en la enseñanza y reconocer los procesos motivacionales subyacentes en las respuestas de los alumnos participantes, se realizó un trabajo de tipo exploratorio, solicitando a los alumnos que dieran respuesta a dos cuestionarios. El primero de los cuestionarios, es un cuestionario estandarizado en la facultad, que deben responde todos los alumnos al final el cursado de una asignatura y busca evaluar la propuesta de enseñanza-aprendizaje ofrecida por la cátedra (desempeño de los docentes, clases, contenidos trabajados, materiales, recursos utilizados, evaluaciones, entre otras variables) El segundo de los cuestionarios fue diseñado de manera específica para obtener información sobre la experiencia, a partir de preguntas cerradas y abiertas que buscaban identificar las razones acerca de por qué habían elegido jugar/no jugar así como la percepción de los alumnos sobre la experiencia. Previa aplicación de dicho cuestionario, se solicitó la revisión del mismo por parte de tres expertos.

\section{Análisis de datos}

Tal como se señaló anteriormente, al finalizar el cursado de la materia, en el año 2014, se les solicitó a los alumnos que respondieran una encuesta en la que se indagó sobre diferentes variables del proceso de enseñanza-aprendizaje llevado a cabo desde la cátedra. En una de las preguntas se pedía a los alumnos que calificaran, en una escala de 1 (Malo) a 5 (Excelente), la propuesta de trabajo con el Juego Kokori.

Como se muestra en el gráfico $\mathrm{N}^{\mathrm{0}} 1$, sobre un total de 137 alumnos que dieron respuesta a dicha encuesta, el $48 \%$ de ellos califica la propuesta como muy buena o excelente, pero al mismo tiempo, hay un porcentaje considerable de alumnos (20\%) que la califican como mala.

Gráfico N$^{\circ}$ 1: Opinión de los alumnos sobre la propuesta de trabajo con Juego Kokori.

Kokori, un serious game. La perspectiva de los estudiantes ante una propuesta de aprendizaje innovadora. 

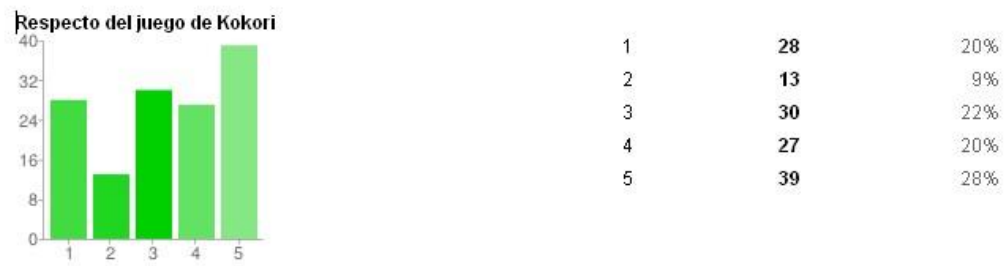

Como complemento de la misma y con el objetivo de conocer la experiencia de aprendizaje vivenciada por los estudiantes con el juego, se realizó una encuesta específica sobre esta actividad del Juego Kokori. Con esta encuesta se buscaba conocer las razones por las cuales los alumnos habían ingresado a jugar el juego y que compartieran su apreciación sobre el mismo; sobre todo si creían que había contribuido en su proceso de aprendizaje y si consideraban conveniente replicar este tipo de actividades en otros contenidos de los trabajados en la asignatura.

$\mathrm{Al}$ acceder a las respuestas de los estudiantes se pudo observar, en primer lugar, que tal como se muestra en el gráfico $\mathrm{N}^{\mathrm{o}} 2$ sobre un total de 163 alumnos que dieron respuesta a la misma, el 67\% (110 alumnos) señalan haber realizado la actividad de jugar al juego de Kokori, mientras que un $33 \%$ de ellos no realizó dicha tarea.

Gráfico $N^{\circ}$ 2: Realización o no de la actividad de jugar al Juego Kokori.

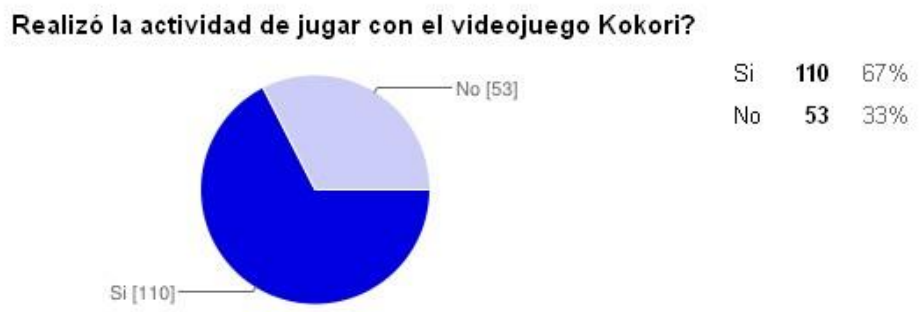

Se analizaron en primer lugar las respuestas de los alumnos que no realizaron la actividad de jugar, para pasar luego a analizar las respuestas de quienes eligieron hacer esta tarea. En el gráfico $\mathrm{N}^{\circ} 3$ se presentan las respuestas de aquellos alumnos que no realizaron la actividad de jugar ${ }^{4}$. Focalizando la atención en este grupo de respuestas, se puede observar que la mayoría de ellos no lo hicieron por razones que parecen estar asociadas a dificultades técnicas y/o de accesibilidad tecnológica. En este sentido los alumnos expresan que "no sabían cómo instalar el juego" (37\%) o bien "no tenían computadora con conexión a internet" (33\%).

En un porcentaje menor, sus respuestas parecen estar asociadas a falta de iniciativa para solicitar los recursos existentes en la facultad y de interés por realizar la actividad; en este sentido los alumnos señalan que "no se animaron a utilizar las máquinas de la facultad” (7\%) o "no les interesó” (3\%).

Otro grupo de razones, en un porcentaje menor, parece estar asociado a desconocimiento. Un grupo de ellos señala que "no sabían que había horarios

\footnotetext{
${ }^{4}$ La pregunta “¿Por qué no jugó?” permitía la posibilidad de múltiples respuestas. Por esta razón, el valor absoluto de la suma de respuestas no coincide con el valor absoluto de alumnos que no realizaron la actividad de jugar al videojuego.
}

Kokori, un serious game. La perspectiva de los estudiantes ante una propuesta de aprendizaje innovadora. M.L. Bossolasco et al. 
disponibles" en la sala de informática de la facultad (7\%) o bien desconocían que se había propuesto dicha actividad desde la cátedra.

Gráfico No 3: Razones por las cuales no jugó al Juego Kokori.
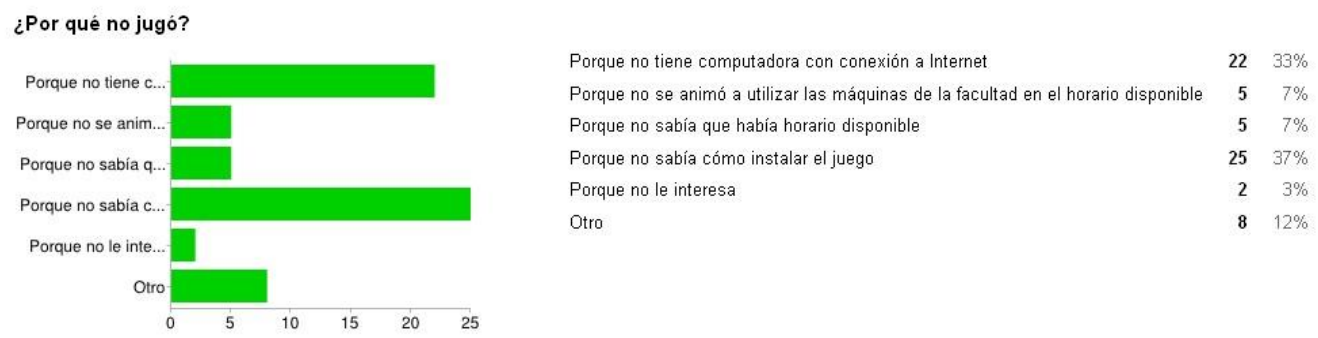

A aquellos alumnos que participaron de la actividad de jugar el videojuego, se les consultó sobre las razones por las cuales eligieron realizar dicha tarea. Pero al mismo tiempo se les solicitó que evaluaran la experiencia en función de dos aspectos; por un lado si creían que la misma había favorecido el aprendizaje de los temas relacionados con el contenido específico (células) y por otra parte, si consideraban que esta experiencia podía resultar útil para el aprendizaje de otros temas. A continuación se presentan las respuestas dadas a estas tres cuestiones.

Tal como se muestra en el gráfico $\mathrm{N}^{\circ}$ 4, entre las razones por las cuales los alumnos participaron $^{5}$ de la experiencia, se evidencian tres grupos de respuestas, con valores próximos. En primer lugar, se ubica un grupo mayoritario de alumnos que participó "para obtener un beneficio en el cursado (ganar un evaluativo)" (31\%), en segundo lugar los alumnos señalan que han participado "por curiosidad" (29\%) y con un porcentaje próximo, otro grupo señala que lo hizo "porque les gusta participar de nuevas experiencias" $(22 \%)$.

Un porcentaje menor de respuestas, aunque también con un valor significativo, señalan que lo hicieron "porque les gusta jugar" (16\%).

Gráfico No 4: Razones por las cuales jugó al Juego Kokori.
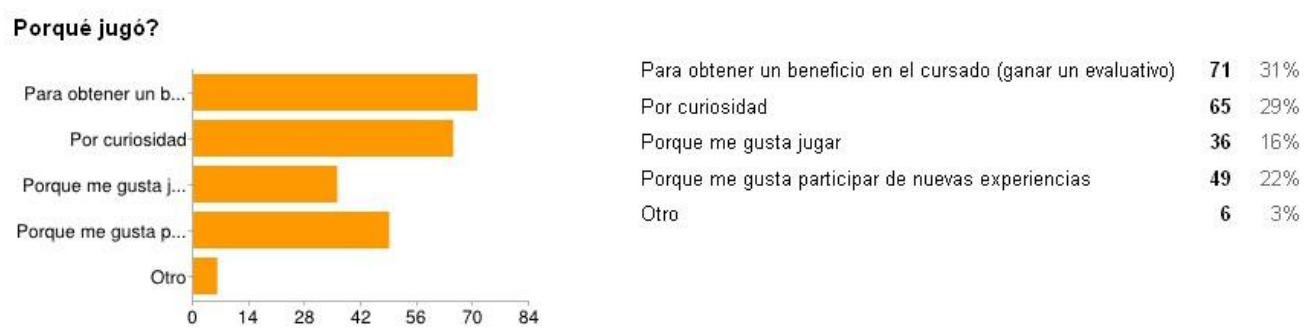

$\mathrm{Al}$ consultar con los alumnos si creen que esta actividad ha favorecido el aprendizaje de los temas relacionados con células, la mayoría de ellos señala que desde su experiencia, sí los ha favorecido. Se puede observar en el gráfico que se muestra a continuación que un $45 \%$ de los alumnos están totalmente de acuerdo y otro $31 \%$ estarían de acuerdo con dicha afirmación. Es considerablemente menor el porcentaje de alumnos que señala valores negativos en dicha respuesta $(5 \%)$.

Gráfico $\mathbf{N}^{\circ} 5$ : Opinión de los alumnos sobre la experiencia de juego como favorecedora de aprendizajes.

\footnotetext{
${ }^{5}$ La pregunta “¿Por qué jugó?” permitía la posibilidad de múltiples respuestas. Por esta razón, el valor absoluto de la suma de respuestas no coincide con el valor absoluto de alumnos que realizaron la actividad de jugar al videojuego. Un mismo alumno podía optar por más de una razón.
}

Kokori, un serious game. La perspectiva de los estudiantes ante una propuesta de aprendizaje innovadora. M.L. Bossolasco et al. 


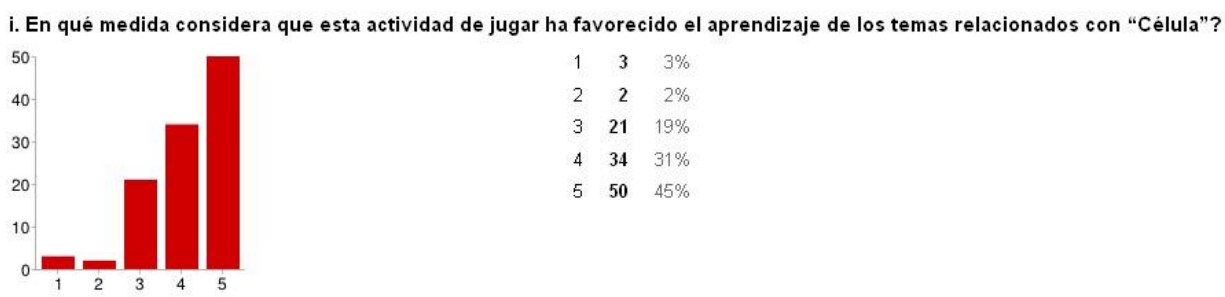

Por su parte, cuando se indaga si consideran que sería conveniente replicar este tipo de experiencias para el aprendizaje de otros temas, se observan ciertas variaciones. Tal como se observa en el gráfico $\mathrm{N}^{\circ} 6$, si bien sigue siendo mayoritario el número de alumnos que responde de manera favorable $38 \%$ (valor 5 de la escala likert) y $28 \%$ (valor 4 de dicha escala); un $16 \%$ de ellos señala que no consideran conveniente replicar dicha experiencia. En este punto, es importante señalar que si bien no se registra en la presenta encuesta, los estudiantes expresaron oralmente que este tipo de actividades resultó interesante para realizar aprendizajes, pero que este juego en particular no podría replicarse en todos los contenidos de la materia; es decir, considera que no resultaría de utilidad para el aprendizaje de otros temas de la asignatura.

Gráfico $\mathbf{N}^{\circ}$ 6: Percepción de los alumnos sobre la posibilidad de replicar la experiencia a otros temas.

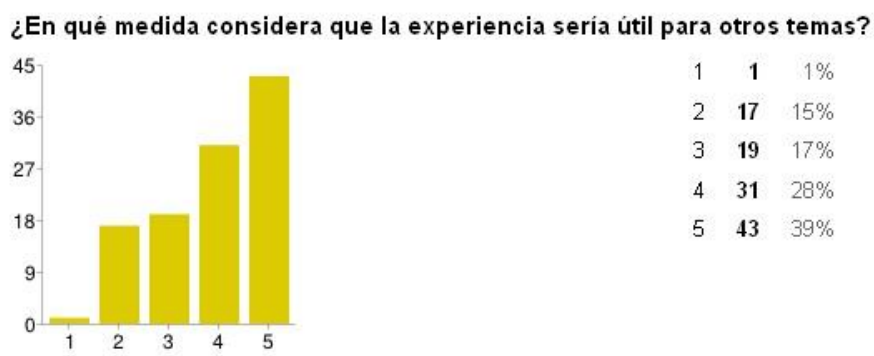

Además de conocer las razones por las cuales realizaron la actividad del videojuego, se les solicitó a los estudiantes que emitieran su opinión sobre la experiencia. Esta pregunta no era obligatoria. La respondieron 76 alumnos, quienes representan el $69 \%$ del total de estudiantes que realizaron la actividad del videojuego.

Entre las opiniones emitidas, existe diversidad de respuestas. Las mismas fueron agrupadas en categorías, surgidas a partir de ciertas recurrencias. Se presentan a continuación dichas categorías, realizando una descripción de las mismas y se incluyen algunas respuestas que permiten ejemplificar.

- Respuestas que evidencian meta-análisis de aprendizajes logrados: Se incluyen aquí aquellas respuestas en donde los participantes hacen referencia explícita a los aprendizajes que realizaron por medio del juego. Para incluir las respuestas en esta categoría se tomaron dos indicadores, por un lado la referencia a la persona del participante ("me ayudó", "me sirvió", "aprendí) y por otro la explicitación acerca del aprendizaje logrado. Estas respuestas fueron, en 
cantidad, las más numerosas, representando un total del $20 \%$ de las opiniones dadas. Algunos ejemplos son:

"A mí personalmente me gustó mucho porque me ayudó a dimensionar la complejidad y la interacción entre los diferentes componentes de una célula, lo cual me resultó de gran ayuda al momento de estudiar la misma." (Respuesta $\mathrm{N}^{\mathrm{o}} 11$ )

"Me pareció buena, me sirvió bastante para reconocer la organización dentro de la célula y la ubicación de las organelas, y a la hora del aprendizaje me resulto más fácil relacionar los contenidos." (Respuesta $\mathrm{N}^{\mathrm{o}} 33$ )

"El juego muy bueno, aunque no pude pasar el ultimo nivel, ahora sé cómo funciona una célula y aprendí muchas funciones de las que no tenía idea, como por ejemplo las funciones de lisosomas, ahora las sé, muy interesante y una muy buena manera para aprender. Me gustó demasiado :D” (Respuesta $\mathrm{N}^{\mathrm{o}} 43$ )

- Respuestas que califican al juego, de manera general, como Muy Bueno y Bueno: En esta categoría se incluyen aquellas respuestas de los estudiantes que realizan una calificación del juego, en función de una escala valorativa. Representa, al igual que en el caso anterior, un $20 \%$ de las respuestas. Las mismas se limitan a señalar que la experiencia fue "muy buena", "satisfactoria", "me pareció bastante buena".

- Respuestas que la reconocen como experiencia educativa novedosa y/o interesante: Se incluyen aquí aquellas opiniones en donde se hace referencia a que ha sido una experiencia lúdica, con fines educativos. En general reconocen este fin educativo dentro del juego, pero asociado a una experiencia que resultó interesante y/o agradable. Este grupo de respuestas, representa el 14\% del total de respuestas dadas. A continuación se transcriben algunas de estas expresiones: "La verdad que me gustó mucho el juego y me ayudó a estudiar." "Estuvo muy buena, es una interesante forma de aprender." "Una experiencia muy original, una manera bastante interesante para aprender."

- Respuestas que la asocian a entretenimiento y diversión: En un porcentaje similar (13\%) se encuentran aquellas respuestas de los alumnos en donde opinan sobre la experiencia asociándola a una experiencia divertida y/o entretenida. En algunos casos, complementan con que ha sido gratificante y atrapante. Algunas expresiones de los estudiantes, incluidas en esta categoría, fueron: "Muy entretenido el juego!", "Divertida, entretenida, educativa, muy gratificante!", "Increíble y entretenida muy aconsejable para jugar y divertirse un rato de un modo más didáctico y atrapante."

Otras opiniones de los alumnos, que se presentan con menor recurrencia, hacen referencia a que:

- Debería haber juegos de este tipo para otros contenidos.

- El juego resultó algo complejo al comienzo.

- Resultó una experiencia aburrida.

Dos participantes realizan sugerencias, respecto al juego en sí y respecto al momento del dictado del contenido en que ellos consideran conveniente se debería presentar la 
actividad de videojuego. Otro alumno señala que lo incorporará como recurso en sus clases, cuando realice su práctica docente.

Como una última cuestión a considerar en el análisis de datos, creemos importante señalar que en el registro del aula virtual se puso en evidencia que durante las primeras semanas de la consigna jugaron pocos alumnos y, al acercarse la fecha límite propuesta, que coincidía con el momento de definir el porcentajes de evaluativos necesarios para acceder al parcial, hubo un incremento notorio de alumnos que accedieron al juego y compartieron sus puntajes en el foro propuesto para tal fin. Se deduce que los alumnos jugaron ya que podía constituirse en una oportunidad de contar con un aprobado más.

\section{Discusión de los resultados}

Considerando los aportes teóricos referidos a los aspectos motivacionales relacionados con el aprendizaje, recordemos que los autores (Paoloni y otros, 2006) reconocen el interjuego de una serie de variables que jugarían un papel relevante en los procesos motivaciones de los estudiantes. Algunas de estas variables, harían referencia a factores personales y otras a ciertos aspectos contextuales.

A partir de los comportamientos observados en el aula virtual, y poniendo el foco en las variables personales, se podría señalar que la participación en la actividad de jugar el videojuego Kokori parece haber respondido a metas extrínsecas, las cuales se orientan a obtener un resultado determinado, en este caso, obtener un evaluativo aprobado para contar con más posibilidades de rendir el examen parcial. Parecería ser que los alumnos estuvieron más interesados en evitar una valoración negativa - perder la posibilidad de tener un evaluativo aprobado- que por un interés en incrementar el conocimiento y mejorar la comprensión sobre el tema, lo cual caracteriza a la motivación intrínseca. De hecho, cuando se les consulta a los alumnos las razones por las cuales participaron del juego, un $31 \%$ de quienes dieron respuesta al cuestionario señalan que lo hicieron "para obtener un beneficio en el cursado (ganar un evaluativo)".

Entre aquellos alumnos que no participaron del juego, las explicaciones que ofrecen en su mayoría responderían atribuciones causales externas. Este grupo de estudiantes, parece responsabilizar a factores externos el hecho de no haber participado en la actividad de juego. Si recordamos, en general señalan que "no tenían computadora con conexión a internet" (33\%). Otro grupo de respuestas hace referencia a variables internas (desconocimiento), pero su postura parece estar ubicada en que la provisión de ese conocimiento debería haber venido desde afuera, como si no ellos no hubiesen tenido responsabilidad en proveerse de ese conocimiento o de solicitar la ayuda necesaria para obtener dicho saber. En este sentido señalan que "no sabían cómo instalar el juego" (37\%), "no se animaron a usar las máquinas de la facultad" (7\%), "no sabían que había horarios disponibles en la sala de informática" (7\%) o desconocían que se había propuesto dicha tarea. La proporción de alumnos que hace referencia a atribuciones causales internas, para justificar su no participación, es mucho menor. Estos alumnos señalan de manera explícita que "no les interesó" participar (3\%).

Pasando ahora a analizar las variables contextuales, se podría señalar que la tarea propuesta cumple con varios de los indicadores que, desde los planteos teóricos, influirían en la motivación de los estudiantes. Recordemos que según señalan Paoloni y otros (2006) el tipo de tarea que se les presente, sería un factor clave para promover la motivación intrínseca. Entre estas condiciones. Se podría señalar que la actividad de jugar al videojuego Kokori fue una tarea que cumple con varios de los requisitos allí señalados:

Kokori, un serious game. La perspectiva de los estudiantes ante una propuesta de aprendizaje innovadora. M.L. Bossolasco et al. 
Variedad y diversidad, lo cual ayuda a mantener el interés. En este sentido, un $22 \%$ de los alumnos que respondieron el cuestionario y señalaron haber participado de la experiencia, explicitan que lo hicieron "porque les gusta participar de nuevas experiencias".

$\checkmark$ Resultó novedosa e imprevisible, generando curiosidad en los estudiantes. De hecho un grupo de estudiantes de los que realizaron la tarea explicitaron que participaron del juego "por curiosidad" (29\%). Por otra parte, al analizar las opiniones emitidas por los alumnos respecto al juego, un $14 \%$ de ellos reconocen que les resultó una experiencia educativa novedosa y/o interesante.

$\checkmark$ Implicó a los estudiantes en la fantasía y la ilusión de hacer algo a través de la simulación o juego. Recordemos que un porcentaje de alumnos (16\%) explicitó que participó de esta actividad "porque les gusta jugar".

Es importante reconocer que si bien, el nivel de dificultad de la tarea en lo referido a los contenidos conceptuales específicos (tema célula) parecen haber estado acorde a las posibilidades de aprendizaje de los alumnos ya que quienes participaron no señalaron que las misiones fueran inaccesibles de realizar; la instalación del juego y la lógica del juego en sí resultó algo exótico para algunos alumnos, requiriendo saberes con los que no contaban en el momento de realizar la actividad y que fue percibido como un esfuerzo desproporcionado por ellos. En este sentido un $37 \%$ de los alumnos que no jugaron explicitaron que "no sabían cómo instalar e juego" y otros hicieron referencia a que "resultó algo complejo al comienzo".

Según las respuestas de aquellos alumnos que realizaron la actividad, el haber participado de este serious games parece haber favorecido el aprendizaje de los temas relacionados con célula. Tal como se señaló en el análisis de datos, un $45 \%$ de los alumnos estuvo totalmente de acuerdo con esta afirmación y otro $31 \%$ estuvo de acuerdo. Los alumnos se interesaron en este tipo de actividad en el aprendizaje, lo cual se podría considerar como una alternativa favorable para incluir en las prácticas educativas, en tanto que sintieron un "autodesafío" de pasar los niveles, les pareció una forma novedosa de aprender y les permitió apropiarse de esos contenidos en una modalidad de aprendizaje autónomo.

Entre las cuestiones a considerar, en el diseño de futuras propuestas de actividades de estas características, se encuentran el nivel de conocimientos previos requeridos para la instalación del juego y el desarrollo de habilidades propias de jugar videojuegos, que en este caso parecen haber actuado como limitantes para algunos alumnos. La instalación del juego requería de ciertos conocimientos técnicos y si bien incluyeron tutoriales en donde se explicaba cómo realizar la descarga e instalación del mismo, la actividad se propuso en el medio de un cursado intensivo dentro de una carrera presencial con una incipiente inclusión de recursos tecnológicos, dando por supuesto que todos los participantes sabrían dar respuesta a dicha demanda.

En conexión con este punto, se pudo ver que el juego tiene todas las características de un video juego, (interacción, envío de órdenes por parte del jugador, logro de un objetivo, medio de entretenimiento) es decir, no es un juego intuitivo para cualquier usuario, por lo tanto el que juega debe tener conocimiento, una experiencia avanzada de videogamers o usuario experto de este tipo de recursos, para que la interacción primera con el juego no desanime a jugar. En este caso en particular se requería: Habilidades para mover la nano nave, familiarizarse con los controles en el tiempo de acción pautado en el juego, identificar las misiones y anticipar las acciones y movimientos requeridas en la misma, entre otras.

Kokori, un serious game. La perspectiva de los estudiantes ante una propuesta de aprendizaje innovadora. M.L. Bossolasco et al. 
En este sentido, habría que considerar aquellas cuestiones del contexto que pueden incidir negativamente en la creencia de autoeficacia de los participantes, disminuyendo la motivación y el compromiso para con la tarea. Recordemos que tal como lo sugieren Paoloni y otros (2006) los juicios que una persona tiene acerca de la capacidad para desempeñarse en acciones específicas, a la luz de metas específicas es una variable personal que incide en la disposición para realizar una tarea. Si la persona percibe que sus niveles de autoeficacia no coinciden con lo que se espera de él para dicha actividad, menor será el esfuerzo, la persistencia y el compromiso para con la tarea.

Retomando los primeros apartados del análisis, en la encuesta general que realiza la cátedra sobre diversas variables del proceso de enseñanza llevado a cabo, la experiencia del juego Kokori no había resultado una experiencia de aprendizaje significativa para un grupo considerable de alumnos. Al indagar, de manera más específica sobre dicha propuesta se puede observar que fue una práctica que varios alumnos realizaron motivados por una recompensa extrínseca. Pero al mismo tiempo se evidencia que, a pesar de haber realizado la actividad, motivados por la búsqueda de un resultado, la tarea de jugar parecería haber generado una posterior motivación intrínseca. Entraron para ganar un evaluativo, pero luego de haber vivido la experiencia de aprendizaje la consideran como una experiencia significativa y viable de replicar en el aprendizaje de otros contenidos.

A partir de esta primera experiencia, se abren nuevos interrogantes, sobre la incorporación que los jóvenes hacen de los recursos provenientes del área de las TIC para su aprendizaje, sobre los procesos motivacionales implicados: ¿Por qué jugaron mayoritariamente con el propósito de obtener una recompensa (motivación extrínseca) si ellos mismos señalan que sería un buen recurso para aprender, internalizar, fijar (motivación intrínseca)?, ¿Lo habrían hecho si no hubiesen obtenido una recompensa o si no poseían una valoración útil y/o interesante de la tarea y del resultado a alcanzar?

Por otra parte, se señala que los docentes deben conocer los códigos y los intereses de los jóvenes y que, en términos de Buckingham (2007) es necesario acortar la brecha; pero cuando desde el contexto educativo se les propone a los jóvenes un videojuego (no obligatorio) no todos los alumnos se implican en realizar esta actividad. ¿Estará relacionado a que son dos lenguajes/códigos/prácticas diferentes, que asocian con ámbitos diferentes y por eso no los vinculan?, ¿No se encuentran interesados en que invadamos esos espacios en los que ellos se "encuentran" para compartir otro tipo de contenidos?, ¿Será que se resisten a que "extendamos el aula" más allá del tiempo que ellos tienen previsto destinar a las tareas académicas? Cuestiones para seguir pensando en el rediseño de entornos y de las propuestas de enseñanza-aprendizaje que se presenten.

Fin de la redacción del artículo: Febrero de 2015.

Bossolasco, M.L et al. (2015). Kokori, un serious game. La perspectiva de los estudiantes ante una propuesta de aprendizaje innovadora. RED, Revista de Educación a Distancia. 45(3). Consultado el (dd/mm/aaaa) en http://www.um.es/ead/red/45 


\section{Bibliografía}

Bossolasco, M. (2013) El concepto de entorno mediado de enseñanza aprendizaje. Significados posibles. En En Chiecher, A; Donolo, D y Córica, J. Entornos Virtuales de Aprendizaje. Nuevas perspectivas de estudios e investigaciones. Consultado el 10/10/14 en http://www.editorialeva.net/evya.html

Castells, M. (2003) La era de la información: Economía, sociedad y cultura. Vol 2. Siglo XXI editores. Bs As.

Chiecher, A., Paoloni, P. y Ficco, C. (en prensa) Ingreso a la Universidad en modalidad a distancia. El papel de aspectos motivacionales y cognitivos en la configuración de logros académicos.

Donolo, D. y Rinaudo, M. (Eds.), (2008) MSLQe - MSLQvv. Motivated Strategies Learning Questionnaire. Propuestas para la medición de la motivación y el uso de estrategias de aprendizaje. Río Cuarto, Argentina: EFUNARC.

Esnaola, G., y Revuelta, F. (2010). Videojuegos y aprendizaje: Formación virtual del profesorado. Herramientas colaborativas y desarrollo de contenidos. Actas del X Encuentro Internacional Virtual Educa Argentina 2009. Consultado el 26/06/14 en http://virtualeduca.info/CDISBN/VirtualEduca.swf

Frasca, G. (2009) Juego, videojuego y creación de sentido. Una introducción. Revista $\begin{array}{lllll}\text { Comunicación. } & \mathrm{N}^{\circ} & 7 . & \text { Consultado }\end{array}$ http://www.revistacomunicacion.org/pdf/n7/articulos/a3_Juego_videojuego_y_creaci on_de_sentido_una_introduccion.pdf

Gardner, H; Davis, K. (2014) La generación APP. Cómo los jóvenes gestionan su identidad, su privacidad y su imaginación en el mundo digital. Bs As. Paidós.

Garretón, V., Báez, M., Carpinelli, J., Mercovich, E., Serrano, I., González, M., Szwarberg Brachitta, M. y Gallardo, R. (2014) Videojuego Kokori. Enseñar biología celular jugando. Docentes Innovadores.net. Consultado el 10/10/14 en http://docentesinnovadores.net/Contenidos/Ver/3217

Ito, M. (2009) Engineering Play: A cultural history of children's software. Cambridge. Massachussets Institute of Technology. Consultado el 21/06/14 en: http://www.journalofplay.org/sites/www.journalofplay.org/files/pdf-articles/2-4book-review-8.pdf

Paoloni, P., Rinaudo, M., Donolo, D., y Chiecher, A. (2006). Motivación. Aportes para su estudio en contextos académicos. Editorial de la Fundación Universidad Nacional de Río Cuarto. Río Cuarto.

Paz, M. (2013) Accesibilidad y usabilidad de los recursos educativos. Revista electrónica Aprender para educar con tecnología. Instituto Nacional Superior del Profesorado Técnico. UTN regional Bs As. Consultada el 10/10/14 en http://es.calameo.com/read/0019480191294304d2299 
Pintrich, P., Smith, D., García, T. y McKeachie, W. (1991). A manual for the use of the Motivated Strategies for Learning Questionnaire (MSLQ). Michigan, MI: National Center for Research to Improve Postsecondary Teaching and Learning.

Revuelta, F., y Guerra, J. (2012) ¿Qué aprendo con videojuegos? Una perspectiva de meta-aprendizaje del videojugador. RED, Revista de Educación a Distancia. Número 33. Consultado el 21/06/14 en http://www.um.es/ead/red/33/revuelta.pdf

Segal, A y otros (2012) "Material de lectura: No todo es juego: Sorious games y educación". Videojuegos educativos en el aula. Especialización docente de nivel superior en educación y TIC. Bs As. Ministerio de Educación de la Nación.

Szwarberg, M., Garretón, V., y Gallardo, R. (s/f) Kokori. Manual para el docente. Versión on line. Consultado el 10/10/14 en http://issuu.com/tekit/docs/manualkokori 